\title{
Cerebral Air Embolism Following Thyroid Biopsy
}

\author{
Yun Su Hwang, MD¹, In Hwan Lim, MD', Hyun Goo Kang, MD², and Jin Sung Cheong, MD \\ 'Department of Neurology, Wonkwang University School of Medicine and Hospital, Iksan, Korea \\ ${ }^{2}$ Department of Neurology, Chosun University School of Medicine and Hospital, Gwangju, Korea
}

Background: Cerebral air embolism is defined as symptoms and signs after occlusion of cerebral arteries by intracerebral air bubble influx. There have been reports of many cases during an invasive procedure. Herein, we report a case of air embolism after thyroid biopsy. Case Report: A 70-year-old female visited our emergency room with right hemiparesis and global aphasia after thyroid biopsy. Brain computed tomography (CT) and magnetic resonance imaging revealed cerebral air embolism in the left hemisphere, and neck CT revealed air embolism in the left lower lateral neck and mediastinum. She was diagnosed with cerebral air embolism following thyroid biopsy and was treated with high-flow oxygen supply.

Conclusion: Our case showed a good outcome with brain imaging and clinical symptoms due to immediate diagnosis of cerebral air embolism and adequate treatment with high-flow oxygen therapy in a patient who had presented with neurologic symptoms directly after thyroid biopsy.

J Neurocrit Care 2016;9(1):45-49

Key Words: Cerebral air embolism; Fine needle aspiration cytology; Hyperbaric oxygen therapy

\section{INTRODUCTION}

Cerebral air embolism is defined as symptoms and signs after occlusion of cerebral arteries by intracerebral air bubble influx, which present as hemiparesis, dysarthria, seizure, mental deterioration and many other neurologic symptoms. ${ }^{1,2}$ Cerebral air embolism can result from many causes. Invasive procedures such as brain operation, cardiopulmonary bypass, gastroscopy, bronchoscopy, lung biopsy, central venous catheter insertion and removal are common causes. ${ }^{3,4}$ Less commonly, air embolism can occurs in divers, head and neck trauma or pneumothorax. ${ }^{2}$ Cerebral air embolism needs to be suspected and treated immediately in patients with neurologic dysfunction after invasive procedure which makes complications.

Cases of cerebral air embolism following gastroscopy, per-

Received: October 22, 2015 / Revised: December 14, 2015

Accepted: January 31, 2016

Address for correspondence: Jin Sung Cheong, MD Department of Neurology, Wonkwang University School of Medicine and Hospital, 895 Muwang-ro, Iksan 54538, Korea

Tel: +82-63-859-1410, Fax: +82-63-842-7379

E-mail: karlkarl@wonkwang.ac.kr cutaneous lung biopsy and coronary angiography had been reported, ${ }^{1,2,5}$ but cases after fine needle aspiration cytology (FNAC) of thyroid mass were not reported yet. FNAC is a useful diagnostic tool in the evaluation of masses in the head and neck, which is simple to perform, quick, accurate and cost-effective. Serious complications are uncommon. ${ }^{6}$ Only minor complications such as focal inflammation were noted, but cerebral air embolism was not reported as a complication of FNAC. We report a case of cerebral air embolism following FNAC of thyroid mass.

\section{CASE REPORT}

A 70-years old female arrived at our emergency medical center with right hemiparesis and global aphasia, which had occurred during fine needle aspiration biopsy of thyroid mass. The symptoms occurred in one minute after needle insertion, and the needle removed immediately from her neck. The time when she arrived at our emergency room (ER) was one hour after the onset of her neurological symptoms. Her vital signs showed blood pressure $150 / 90 \mathrm{mmHg}$, respiration rate $20 / \mathrm{min}$, heart rate $63 / \mathrm{min}$, oxygen saturation $98 \%$ and 

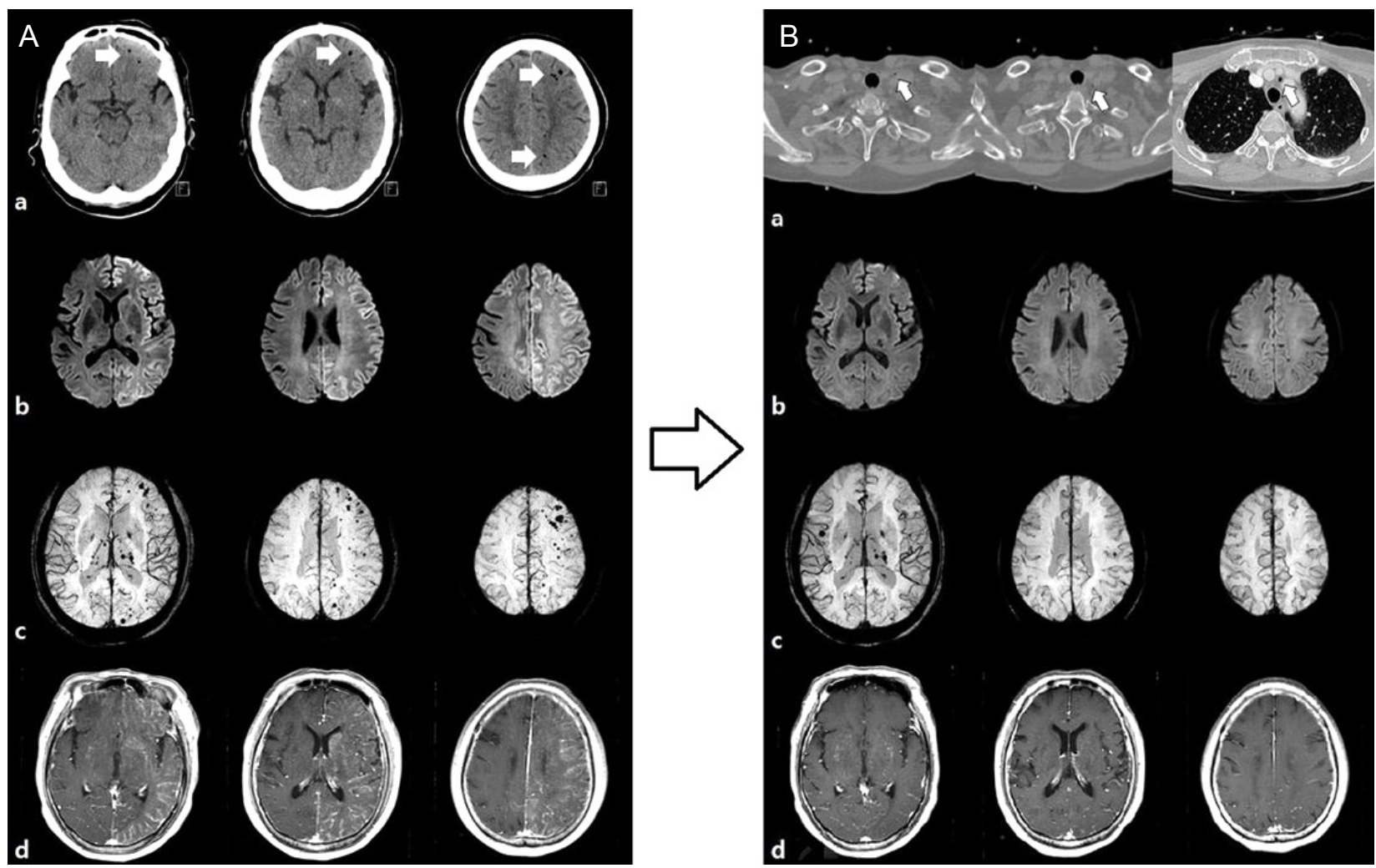

Figure 1. (A) Brain computed tomography (CT) and magnetic resonance image (MRI) demonstrate acute cerebral ischemia with cerebral air embolism. (a) Unenhanced CT shows several hypodense lesions in left cerebral hemisphere, suggesting multiple scattered small air bubbles (white arrows). (b) In diffusion weighted image (DWI), typical diffuse high signal intensity lesions in left entire cerebral hemisphere and basal ganglia are seen. (c) Susceptibility weighted image (SWI) shows multiple scattered air bubbles in mainly left frontal subcortex. (d) Diffuse parenchymal and leptomeningeal enhancement are seen in left cerebral hemisphere suggesting early blood-brain barrier (BBB) disruption. (B) Neck CT reveals small air collection and follow-up magnetic resonance imaging (MRI) at 8 days later demonstrate resolution of cerebral air embolism after treatment. (a) In neck CT, small amount of air collection in left lower lateral neck and superior mediastinum are noted (white arrows). (b, c, d) Brain MRI shows resolution of diffuse ischemic lesions, air embolism and BBB disruption in left entire cerebral hemisphere in DWI, SWI and enhanced T1 images in contrast to previous images.

body temperature $36.2^{\circ}$. EKG revealed nonspecific findings. She didn't have any medication. On neurologic examination, she was alert but left-sided forced eyeball deviation, global aphasia, right hemiparesis and positive-Babinski sign was noted. This symptoms and signs were similar to left middle cerebral artery infarction. Brain computed tomography (CT) was showed multiple scattered air bubbles on the left hemisphere (Fig. 1A-a). Immediately, we started monitoring for vital signs and treatment with high-flow of oxygen supply on diagnosis of cerebral air embolism. Because hyperbaric oxygen therapy could not be adapted in our hospital, we used mask with reservoir bag with $10 \mathrm{~L} / \mathrm{min}$ of oxygen flow, equal value of $\mathrm{FiO}_{2}>0.99$. After initiation of oxygen therapy, brain magnetic resonance imaging (MRI) was performed in serial. Diffusion weighted image (DWI) showed very subtle high signal intensity (HSI) on the subcortex of left hemisphere, but its signal was not quite obvious. Susceptibility weighted image (SWI) showed multiple scattered air bubbles were visible, and T1 weighted enhanced image showed diffuse enhancement in left hemisphere as an early sign of diffuse blood-brain barrier breakdown (Fig. 1A-c, d). In MR angiography, definite occlusion of intracranial vessels were not detected, but in perfusion weighted image (PWI), mild perfusion deficit was seen which was suspected occlusion of distal branches of left middle cerebral artery. Two hours after, she received follow up MRI. DWI showed obvious diffuse HSI was seen in left hemisphere (Fig. 1A-b).

Five hours after arrival to ER, she suddenly showed partial seizure with clonic movement of right hemibody, followed by secondary generalized seizure with generalized tonic clonic movement. Seizure subsided after intravenous injection of lorazepam. Electroencephalogram was performed and showed 
diffuse intermittent slow waves in left hemisphere. We performed neck CT to find origin of cerebral air embolism and found small amount of air collection in left lower lateral neck and superior mediastinum (Fig. 1B-a). We continued oxygen therapy, close monitoring for vital and neurologic signs and general supportive care after admission to intentional care unit. The day after admission, her neurologic symptoms started to improve gradually. As her mental status waked up and complained of hyperventilation due to the oxygen mask with high flow, we gradually tapered the oxygen flow off to 6/ $\mathrm{min}$. At the next day, we changed to simple nasal cannula 3-5 $\mathrm{L}\left(\mathrm{FiO}_{2} 0.4\right)$.

Five days after admission, her motor weakness and aphasia was recovered but mild dysarthria was remained as neurologic sequelae. We stopped oxygen therapy. At eighth hospital day, she received follow up MRI. In DWI, diffuse high signal intensity in left hemisphere, which showed in the previous study, showed almost resolution, which only a few residual parenchymal lesions were marked in fluid attenuated inversion recovery (FLAIR) image. In SWI, air bubbles, which showed in the previous study, also disappeared and in T1 weighted enhanced image, diffuse parenchymal enhancement was resolved, too (Fig. 1B-b, c, d). She was discharged from the hospital at the next day with only mild slurring, but no other neurologic deficit.

\section{DISCUSSION}

There are two broad categories of gas embolism, venous and arterial, which are distinguished by the mechanism of gas entry and the site where the emboli ultimately lodge. ${ }^{7}$ Venous air embolism occurs when a biopsy needle opened to the atmosphere punctures a vein: if the venous pressure decreases under these conditions, as during inspiration, and the atmospheric pressure exceeds the venous pressure, air may enter the pulmonary venous system. ${ }^{5}$ After flow into pulmonary vein, patent foramen ovale (PFO), which is detectable in about 30 percent of the general populations makes possible right to left shunting of gas bubble. ${ }^{6,7}$ So gas bubble flow into systemic circulation, which results in cerebral air embolism. And without PFO, when the amount of air exceeds the lung capacity, physiologic pulmonary vein to systemic artery shunt can makes systemic air embolism. ${ }^{8}$ Arterial gas embolism is caused by the entry of gas into the pulmonary veins or directly into the arteries of the systemic circulation. ${ }^{7}$ In our patient, we suspect that both arterial and venous air embolism were considerable. Within the framework of arterial embolism, we suspect that the needle had been inserted to adjacent left lower neck, not into thyroid tissue due to technical problem, and air bubbles were absorbed into adjacent carotid artery directly. And if it was venous air embolism, it is suspected that air bubbles absorbed into thyroid vein during procedure entered pulmonary vein and systemic circulation via right to left shunt such as possible PFO or physiologic shunt.

Cerebral arterial gas embolization commonly induces the migration of gas to small arteries (average diameter, 30-60 $\mu \mathrm{m})$. The air bubbles causes a reduction in perfusion distal to the obstruction, makes cytotoxic edema and inflammatory response to the bubble. ${ }^{2,7}$ Signs and symptoms of cerebral air embolism resembles that of acute ischemic stroke, it means focal neurologic deficit related to involved brain lesion can be seen. Our patient's symptoms were similar to that of acute ischemic stroke in left middle cerebral artery territory, because cerebral air embolism had involved left middle cerebral artery, which were presented with global aphasia, right hemiparesis and facial palsy, and forced eyeball-deviation to left side.

At brain CT, air embolism can be detected as a round shaped hypodense lesion and damaged brain tissues also are more hypodense than other normal tissues. But air embolisms dissolve in blood so fast that air bubbles can be only seen in early stage and disappear soon. ${ }^{1,9}$ At brain MRI (DWI), even in patients with obvious air embolism, any significant findings can not be seen in very early stage, therefore air embolism can not be excluded with negative DWI. ${ }^{8,10}$ In suspected patients with negative DWI, retry of DWI is recommended.

The most important treatment of cerebral air embolism is hyperbaric oxygen (HBO) therapy. ${ }^{1,5,711} \mathrm{HBO}$ therapy is supplying $100 \%$ oxygen to a patient at high pressure. The preferred therapy is 2.5-3.0 atmospheres for 2 to 4 hours, with repeated or longer treatment until symptom free or no further clinical improvement. ${ }^{11}$ There are several principles that explain the rationale behind HBO therapy. The first is Boyle's law. By decreasing the size of an already formed bubble in the circulation by placing the patient in a high pressure environ- 
ment, mechanical obstruction can be overcome. The second important principle is Henry's law: This implies that if oxygen is given at high pressure the amount of oxygen dissolved in plasma will increase. By increasing pressure and concentration of oxygen in inspired air the amount of dissolved oxygen in plasma increases proportionately. This helps in increasing oxygen delivery to the tissues as fluid with dissolved oxygen can seep through the mechanical obstruction that holds red blood cells and hemoglobin. Thirdly, by breathing oxygen rich and nitrogen poor gas the concentration of nitrogen in the blood falls, which draws nitrogen gas in embolized bubbles back into plasma in soluble form, further contributing to decrease in size of air bubbles. Fourthly, HBO can induce arterial vasoconstriction and decrease permeability of the blood-brain barrier, thereby preventing cerebral edema. It also decreases the adherence of leucocytes and thereby potential damage to the endothelium. ${ }^{5}$ HBO therapy must be initiated as soon as possible from occurrence of the air embolism. The shorter time interval from occurrence, the better prognosis of patients can be expected. In fact, our patient did not received hyperbaric oxygen therapy and were treated with normobaric high-flow oxygen therapy with reservoir bag mask due to unavailability of $\mathrm{HBO}$ equipment. But not only HBO therapy but also normobaric high-flow oxygen therapy can improve the prognosis of air embolism. As HBO therapy does, high flow oxygen also can increase ther partial pressure of oxygen and decrease the partial pressure of nitrogen. It results in reducing bubble size and inducing bubble resorption through diffusion of nitrogen from air bubble in to the blood. Although the optimal $\mathrm{FiO}_{2}$ is unknown, the highest level of $\mathrm{FiO}_{2}$ to achieve highest oxygen saturation should be considered. In the absence of available HBO therapy equipment, if the patient can tolerate, High flow of oxygen therapy with $\mathrm{FiO}_{2}$ 1.0, often with the aid of a ventilator is recommended for cerebral air embolism. ${ }^{12,13}$

With oxygen therapy, other supportive care also should be performed with close monitoring for vital signs. Supportive measures involve managing seizure activity, proper patient positioning, intubation if airway protection is needed and fluid hydration. ${ }^{5,711}$

Fluid resuscitation is also important. Hemoconcentration directly caused by air embolism increased blood viscosity and impairs the microcirculation in the ischemic area. Fluid hy- dration can decreased the hemoconcentration. Rehydration further allows microcirculation to the areas of impairment. Colloid solutions are preferred over crystalloid, as the latter may worsen cerebral edema. The goal of infusion therapy is normovolemia. Placement of a central venous catheter to assess central venous pressure and Foley catheter insertion for monitoring of urinary output are recommended. ${ }^{7,11}$ Therefore, the most important treatment for cerebral air embolism is immediate initiation of $\mathrm{HBO}$ therapy with supportive care for vital signs. ${ }^{14}$

Our patient showed improvement of neurologic symptoms dramatically with immediate treatment including oxygen therapy and generalized supportive care. In fact, the prognosis of cerebral air embolism is generally poor and often makes serious neurologic sequelae for a long time. But immediate diagnosis and treatment can make good outcome like our patient.

\section{REFERENCES}

1. Song DB, Cho HJ, Ye BS, Heo JH. Cerebral air embolism following a gastroscopy. J Korean Neurol Assoc 2008;26:146-8.

2. Oh HJ, Kwon SB, Kim MJ, Hong SS, Minn YK, Jung S, et al. Acute middle cerebral artery infarction by cerebral air embolism during coronary angiography. J Neurocrit Care 2013;6:122-5.

3. Murphy BP, Harford FJ, Cramer FS. Cerebral air embolism resulting from invasive medical procedures. treatment with hyperbaric oxygen. Ann Surg 1985;201:242-5.

4. Han SS, Kim SS, Hong HP, Lee SY, Lee SJ, Lee BK. Massive paradoxical air embolism in brain occurring after central venous catheterization: a case report. J Korean Med Sci 2010;25:1536-8.

5. Hare SS, Gupta A, Goncalves AT, Souza CA, Matzinger F, Seely JM. Systemic arterial air embolism after percutaneous lung biopsy. Clinical radiology 2011;66:589-96.

6. Ghosh S, Panarese A, Parker AJ. Monocular blindness following fine needle aspiration cytology of a neck mass. J Laryngol Otol 2000;114:712-3.

7. Muth CM, Shank ES. Gas embolism. N Engl J Med 2000;342:47682.

8. Yu SB, Ryu SJ, Yoon SH, Kang HS, Kim DS. Cerebral air embolism after removal of internal jugular venous catheter. Korean $J$ Anesthesiol 2009;57:673-7.

9. Oatis K, Agarwal A, Bruce-Tagoe C. Acute stroke from air embolism to the middle cerebral artery following upper gastrointestinal endoscopy. Radiol Case Reports 2010;5:359.

10. Sayama T, Mitani M, Inamura T, Yagi H, Fukui M. Normal diffusion-weighted imaging in cerebral air embolism complicating angiography. Neuroradiology 2000;42:192-4. 
11. Pandurangadu AV, Paul JA, Barawi M, Irvin CB. A case report of cerebral air embolism after esophagogastroduodenoscopy: diagnosis and management in the emergency department. J Emerg Med 2012;43:976-9.

12. Sood B, Rezaei S, Brodribb R. Iatrogenic air embolism following CT guided lung biopsy. Appl Radiol 2012;41:43-44,46

13. Annane D, Troche G, Delisle F, Devauchelle P, Paraire F, Raphael
JC, et al. Effect ofmechanical ventilation with normobaric oxygen therapy on the rate of air removal from cerebral arteries. Crit Care Med 1994;22:851-7

14. Blanc P, Boussuges A, Henriette K, Sainty JM, Deleflie M. Iatrogenic cerebralair embolism: importance of an early hyperbaric oxygenation. Intensive Care Med 2002;28:559-63. 